\title{
Biological predictors of suicidality in schizophrenia
}

Lewis CF, Tandon R, Shipley JE, DeQuardo JR, Jibson M, Taylor SF, Goldman M. Biological predictors of suicidality in schizophrenia. Acta Psychiatr Scand 1996: 94: 416-420. (c) Munksgaard 1996.

The objective of this study was to determine whether polysomnographic rapid eye movement (REM) sleep abnormalities and cortisol response to the dexamethasone suppression test (DST) differentiate between schizophrenic patients with and without a history of suicidal behaviour. We assessed a sample of 96 schizophrenic in-patients at the end of a 2-week medication-free period with the DST, polysomnography, and an extensive clinical assessment battery. Patients exhibiting suicidal behaviour were significantly more likely to have increased total REM time and increased total REM activity. We found no significant relationship between suicidal behaviour and DST non-suppression. This study confirms a previous finding suggesting an association between REM sleep abnormalities and suicidal behaviour in schizophrenia. It is postulated that this observed association may be related to serotonergic dysfunction in schizophrenia.

\section{F. Lewis, R. Tandon, J. E. Shipley, J. R. DeQuardo, M. Jibson, S. F. Taylor, M. Goldman}

Schizophrenia Division, University of Michigan Medical Center, Ann Arbor, MI, USA

Key words: schizophrenia; suicidality polysomnography; dexamethasone suppression test; REV sleep

Rajiv Tandon, Schizophrenia Program, University of Michigan Medical Center, Ann Arbor, MI 48109-0116, USA

Accepted for publication April 13, 1996

\section{Introduction}

Suicidal behaviour is a significant complication of schizophrenic illness. Approximately $50 \%$ of schizophrenic patients seriously consider or attempt suicide, and approximately $10 \%$ eventually succeed (1). Numerous clinical predictors of suicidal behaviour in schizophrenic patients have been described, including young age, early stage of illness, substance abuse, college education, multiple episodes of psychosis, living alone, previous suicide attempts, depression/hopelessness, and stress (2-8). Recent research in the field has focused on the neurobiological substrates of suicidal behaviour in schizophrenia; as yet, this area remains largely undefined.

Investigators have sought to draw lessons from the substantial body of research conducted on the pathophysiology of suicidal behaviour in major depression. Studies of cortisol secretion following administration of dexamethasone suggest that 40$50 \%$ of patients with major depression exhibit cortisol non-suppression (9), and that cortisol nonsuppression is significantly more frequent in depressed suicidal patients than in depressed nonsuicidal patients (9). Suicidal behaviour in patients with major depression has also been associated with sleep abnormalities (10). Sabo et al. studied a group of patients with depression and found that, compared to non-attempters, suicide attempters exhibited increased sleep latency, decreased sleep efficiency, increased total REM time and increased REM activity.

Although cortisol non-suppression of the DST and REM sleep abnormalities were originally considered to be abnormalities specific to major depressive disorder, recent research has clearly documented both types of abnormalities in a substantial proportion of schizophrenic patients $(11,12)$. Reports of an association between both REM sleep and DST abnormalities and suicidal behaviour in schizophrenia have recently been published $(13,14)$. Keshavan et al. studied a sample of 19 schizophrenic subjects and reported that patients exhibiting suicidal behaviour had significantly increased overall REM activity and REM time (13). Jones et al. found that non-suppression of the DST may be related to suicidal behaviour in a sample of 57 schizophrenics; specifically, nonsuppression of the DST differentiated suicide attempters from non-attempters (14). While these findings are obviously significant, they are of a preliminary nature. The aim of the present study was to evaluate the relationship between REM sleep abnormalities, DST response and suicidal 
behaviour in a large sample of schizophrenic patients.

\section{Material and methods}

Subjects

The study sample consisted of 96 patients who were admitted to the Schizophrenia Program at the University of Michigan. All of the patients received an extensive diagnostic evaluation, including a structured interview using the Schedule for Schizophrenia and Affective Disorders (SADS) (15), and fulfilled both Research Diagnostic Criteria (RDC) (16) and DSM-III-R (17) criteria for schizophrenia. Patients with any medical condition known to interfere with a valid DST (9), and with any known ongoing drug or alcohol use were excluded from the study. All patients were medication-free for a minimum of 2 weeks, and none of them had received depot neuroleptics in the preceding 6 months. The mean age $( \pm S D)$ of all patients was $29 \pm 9.2$ years, and the sample consisted of 66 male and 30 female subjects.

At medication-free baseline, all patients were rated on the 18-item Brief Psychiatric Rating Scale (BPRS) (18), the Scale for the Assessment of Negative Symptoms (SANS) (19) and the 17-item Hamilton Depression Rating Scale (HDRS) (20). Global severity was assessed by means of the 18item BPRS total score. Positive symptom severity was assessed as the sum of the following four BPRS items: conceptual disorganization, suspiciousness, hallucinatory behaviour and unusual thought content (21). Negative symptoms were quantified by the SANS sum of global scores. Other relevant sociodemographic and clinical variables used to characterize the sample included full-scale IQ, age at first hospitalization, and number of years of education.

\section{Methods}

Lifetime history of suicidal behaviour was obtained from a review of the clinical and research records of each patient by a rater (C.F.L.) who was blind to the DST and sleep data. Assessment involved extensive chart review, and review of the SADS interview and 1-year follow-up data. Suicidal behaviour was defined on a scale which ranged from 0 to 2 , where 0 denoted patients with no suicidal ideation or attempts (1-2 on SADS item no. 246), 1 denoted patients with suicidal ideation (3-4 on SADS item no. 246) and 2 denoted patients with suicide attempts (5-7 on SADS item no. 246); SADS ratings were corroborated by case records from the clinical charts.
A 1-mg DST (9) was performed at baseline. Blood samples for post-dexamethasone cortisol levels were obtained at 16.00 and 23.00 hours, and the maximum cortisol levels were used for analysis. Cortisol levels were assayed by Murphy's competitive protein-binding method (22). Maximal postdexamethasone cortisol levels greater than $5 \mu \mathrm{g}$ $\mathrm{dl}^{-1}$ have been shown to be indicative of nonsuppression (9). Details of the DST methodology are described in Tandon et al. (12). Variables utilized for analysis included (a) maximal postdexamethasone cortisol levels and (b) status as DST non-suppressor or suppressor.

Two-night polysomnography was conducted in the patient's hospital beds as described by Tandon et al. (11). The first night was used to exclude any underlying sleep abnormalities and the second night was used for data analysis. Patients were assessed for primary sleep disorders by means of questionnaires, and were excluded from the study if such abnormalities were noted. Manually derived sleep measures were obtained by technicians (who were blind to the clinical data on sleep) at 60-s intervals using the criteria of Rechstaffen and Kales (23). Relevant sleep measures used for analysis included sleep latency, total number of minutes asleep, time and percentage of sleep spent in Stages 1 to 4 NREM and REM, and several REM measures, including REM latency, activity and density.

\section{Data analysis}

The relationship between suicidal behaviour and sleep, clinical and DST data was evaluated by Kendall's rank-correlation analysis. Stepwise regression between these variables was then performed in order to identify predictors of suicidal behaviour while controlling for other variables. Suicidal behaviour was the dependent variable, and clinical variables and sleep and DST data were the independent variables.

\section{Results}

There were 36 patients in group 1 (no suicidal ideation/attempts), 38 patients in group 2 (suicidal ideation but no attempts) and 22 patients in group 3 (suicidal attempts). Patients who exhibited suicidal behaviour had increased total REM time $(r=0.40 ; P<0.001)$ and increased REM activity $(P<0.05)$ (Fig. 1). However, no correlation was found between suicidal behaviour and proportion of slow-wave sleep (SWS), or between suicidal behaviour and REM latency, neither was there any significant correlation between baseline postdexamethasone cortisol levels and suicidal behaviour (Table 1). 


\section{Lewis et al.}

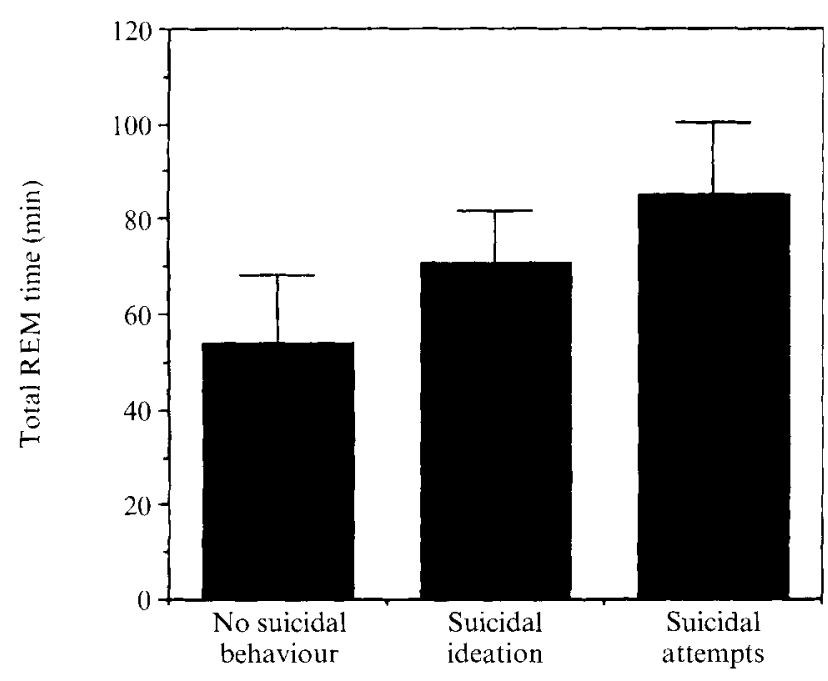

Fig. 1. Suicidal behaviour and total REM time in schizophrenia; $n=78$. Mean values \pm SD are shown.

Table 1. Clinical, DST and REM sleep correlates of suicidal behaviour in schizophrenia

\begin{tabular}{|c|c|c|c|}
\hline \multirow[b]{2}{*}{ Variable } & \multicolumn{3}{|c|}{ Rank-order correlation } \\
\hline & $\begin{array}{l}\text { Correlation with } \\
\text { suicidal behaviour } \\
\text { (tau value) }\end{array}$ & $n$ & $P$-value \\
\hline \multicolumn{4}{|l|}{ REM sleep } \\
\hline REM latency & -0.01 & 78 & NS \\
\hline Total REM \{min\} & 0.40 & 78 & $<0.001$ \\
\hline REM activity & 0.16 & 78 & $<0.05$ \\
\hline SWS $(\%)$ & -0.02 & 78 & NS \\
\hline \multicolumn{4}{|l|}{ DST data } \\
\hline \multicolumn{4}{|c|}{ Baseline post-dexamethasone cortisol } \\
\hline level & -0.09 & 57 & NS \\
\hline \multicolumn{4}{|l|}{ Clinical variables } \\
\hline Age & -0.13 & 96 & $<0.06$ \\
\hline Gender & -0.22 & 96 & $<0.05$ \\
\hline Level of education & 0.11 & 96 & $<0.10$ \\
\hline \multicolumn{4}{|l|}{ Symptoms } \\
\hline Positive symptoms & -0.05 & 96 & NS \\
\hline Negative symptomis & 0.10 & 96 & NS \\
\hline Global severity (total BPRS) & 0.13 & 96 & $<0.06$ \\
\hline Depression (total HRSD) & 0.19 & 96 & $<0.01$ \\
\hline
\end{tabular}

When the group of suicide attempters was divided into those who had made an attempt in the previous 6 months $(n=6)$ and those who had made no attempts in the previous 6 months $(n=16)$, no difference in any sleep measure or in DST data was noted. Furthermore, when this group of 22 suicide attempters was divided into those with a history of a violent suicide attempt $(n=5)$ and those with no such history $(n=17)$, no difference in sleep or DST findings was observed.
There was a trend for patients who exhibited suicidal behaviour to be of lower age, male and to have received a higher level of education. Positive and negative symptoms were not predictors of suicidal behaviour, but HRSD scores were correlated with suicidal behaviour.

On stepwise regression analysis, two variables emerged as significant predictors of suicidal behaviour, namely total REM sleep $(r=0.40 ; P<0.001)$ and patient gender $(r=0.50 ; P<0.001)$. Together, these two variables accounted for $25 \%$ of the total variance in suicidal behaviour.

\section{Discussion}

This study found no significant correlation between suicidal behaviour and post-dexamethasone suppression levels. This result is in contrast to that of Jones et al. (14), and is particularly striking given the high rate of dexamethasone non-suppression $(40 \%)$ observed in this sample of schizophrenic patients. DST non-suppression has been associated with shortened REM latency in depressive subjects (24), and it is salient that neither DST nonsuppression nor decreased REM latency were found to be associated with lifetime suicidal behaviour in the present study. This suggests that the mechanisms underlying DST non-suppression and shortened REM latency in schizophrenia may be state-related (25), and unrelated to suicidal behaviour. Our observation of an association between suicidal behaviour and male gender, longer education and younger age is consistent with previous reports of clinical predictors of suicidal behaviour in schizophrenia.

The main positive finding in this study is the significant association between total REM sleep time and suicidal behaviour. Total REM activity was also found to be significantly correlated with suicidal behaviour, a finding that is consistent with previous studies of schizophrenia (13). These results are similar to the data for suicidal patients with major depression (10). It is noteworthy that depression severity scores as assessed by the HRSD did not correlate significantly with REM sleep abnormalities (11). The significant association between total REM sleep time and suicidal behaviour suggests that a common mechanism may underlie both of these abnormalities in schizophrenia. Although the precise nature of this common mechanism is unknown, reduced central nervous system (CNS) serotonergic function, as measured by 5-hydroxy indole-acetic acid (5HIAA) levels in the cerebrospinal fluid, is associated with suicidal behaviour in depression $(10,26,27)$ and schizophrenia $(13,28)$. Since serotonergic neurones act to suppress REM sleep, 
reduced serotonergic function in schizophrenia could explain the association between suicidal behaviour and REM time/activity observed in the present study. Serotonergic hypoactivity has been specifically implicated in violent suicide attempts $(29,30)$. However, we observed no significant difference between violent and non-violent suicide attempters in our sample of schizophrenic patients.

It should be emphasized that schizophrenic illness in general is not characterized by any abnormality of REM time or activity (11). However, within the group of schizophrenic patients, subjects with longer REM time and higher REM activity are more likely to exhibit lifetime suicidal behaviour than those with shorter REM time and lower REM activity. Thus longer REM time and higher REM activity might be a marker of suicidal behaviour in schizophrenia. However, this association does not appear to be specific to schizophrenia, as it has also been described in samples of depressed patients (10).

The association between total REM sleep and activity and suicidal behaviour is somewhat surprising, given the fact that the sleep studies were conducted during a state of acute psychotic exacerbation, while lifetime suicidal behaviour reflects a trait abnormality in schizophrenia. When we compared recent suicide attempters (attempts within the previous 6 months) with those with a lifetime history of suicide attempts but without a history of recent attempts, we observed no significant difference between the groups with regard to REM time or activity, or any sleep or DST findings. However, REM time and activity are relatively stable throughout a course of schizophrenic illness. Although they were assessed during a psychotic state, they may in fact reflect a stable trait feature, and this might explain the association between REM time and lifetime history of suicidality that we observed in our sample of schizophrenic patients.

Caution must be exercised when relating possible state findings, such as sleep data, to trait aspects, such as lifetime history of suicidal behaviour. However, its documentation in two separate sets of data suggests that it merits further study.

\section{References}

1. Roy A. Depression, attempted suicide and suicide in patients with chronic schizophrenia. Psychiatr Clin North Am 1986: 9: 193-206.

2. Allebeck P, Varla A, Kristjansson Wistedt B. Risk factors for suicide among patients with schizophrenia. Acta Psychiatr Scand 1987: 76: 414-419.

3. Drake GE, Gates C, Whitaker A, Cotton PG. Suicide among schizophrenics: a review. Compr Psychiatry 1985: 26: $90-100$.
4. Nyman AK, Jonsson SH. Patterns of sclf-destructive behaviour in schizophrenia. Acta Psychiatr Scand 1986: 73: $252-262$.

5. Dassori AM, Mezzick JE, Keshavan M. Suicidal indicators in schizophrenia. Acta Psychiatr Scand 1990: 81: 409-413.

6. Addington DE, Addington JM. Attempted suicide and depression in schizophrenia. Acta Psychiatr Scand 1992: 85: $288-291$.

7. Modestin J, Zarro I, Waldvoghi. D. A study of suicide in schizophrenic inpatients. Br J Psychiatry 1992: 160: 398-401.

8. Landmark J, Cernovsky ZZ, Merskey H. Correlates of suicide attempts and ideation in schizophrenia. $\mathrm{Br}$ J Psychiatry 1987: 151: 18-20.

9. Carroll. BJ, Greden JF, Feinberg M. Suicide, neuroendocrine dysfunction and CSF 5-HIAA concentrations in depression. Recent Adv Neuropsychopharmacol 1981: 31: 307-313.

10. Sabo E, Reynolds CF, Kupfer DJ, BFrman SR. Sleep, depression and suicide. Psychiatry Res 1990: 36: 265-277.

11. Tandon R, Shipley JE, Taylor SF et al. Electroencephalographic sleep abnomalities in schizophrenia: relationship to positive/negative symptoms and prior neuroleptic treatment. Arch Gen Psychiatry 1992: 49: 185-194.

12. Tandon R, Mazzara C, DeQuardo JR et al. Dexamethasone suppression test in schizophrenia: Relationship to symptomatology, ventricular enlargement and outcome. Biol Psychiatry 1991: 29: 953-964.

13. Keshavan MS, Reynolds CF, Monitrose D. Miewai.d J. Downs C, SABO EM. Sleep and suicidality in psychotic patients. Acta Psychiatr Scand 1994: 89: 122-125.

14. Jones JS, Stein DJ, Stanley B, Guido JR. Wincilel R, Stanley M. Negative and depressive symptoms in suicidal schizophrenics. Acta Psychiatr Scand 1994: 89: 81-87.

15. Endicott J, Spitzer RL. A diagnostic interview: the schedule for Affective Disorders and Schizophrenia. Arch Gen Psychiatry 1978: 35: 837-844.

16. Sprizer RL, Endicotr J, Robins E. Research diagnostic criteria: rationale and reliability. Arch Gen Psychiatry 1978: 35: $773-782$

17. American Psychiatric Association. Diagnostic and statistical manual of mental disorders, revised 3rd edn (DSM-III-R) Washington, DC: American Psychiatric Association, 1987.

18. Overall JE, Gorham DR. Brief Psychiatric Rating Scale. Psychiatric rating scale. Psychol Rep 1962: 10; 799-812.

19. Andreasen NC. The Scale for the Assessment of Negative Symptoms. Iowa City, Iowa: University of lowa, 1983.

20. Hamilton M. Development of a scale for primary depressive illness. Br J Soc Clin Psychol 1960: 6: 278-296.

21. Tandon R, Ribeiro SCM, Dequarbo JR, Goldman RS, GoOdson J, Greden JF. Covariance of positive and negative symptoms during neuroleptic treatment in schizophrenia: a replication. Biol Psychiatry 1993: 34: 495-497.

22. Murphy BE. Some studies of the protein-binding of steroids and their application to the routine micro- and ultra-micro measurement of various steroids in body fluids by competitive protein-binding radioassay. $\mathrm{J}$ Clin Endocrinol Metab 1967: 27: 973-990.

23. Rechstaffen A. Kales A, Bergtr RJ. A manual of standardized terminology, techniques, and scoring systems for sleep stages in human subjects. Washington, DC: US Public Health Service, 1968.

24. LewiS C, TANDON R, TAYlor SF et al. Relationship between DST nonsuppression and shortened REM latency in schizophrenia. Biol Psychiatry 1996: 40: 660-663.

25. Keshavan MS, TANDON R. Sleep abnormalities in 


\section{Lewis et al.}

schizophrenia: pathophysiological significance. Psychol Med 1993: 23: 831-835.

26. Asberg M, Traskman L, Thoren P. 5HiAa in the cerebrospinal fluid: a biochemical suicide predictor? Arch Gen Psychiatry 1976: 33: 1193-1197.

27. Nordstrom P, Samuelsson M, Asberg $M$ et al. CSF 5-HIAA predicts suicide risk after attempted suicide. Suicide Life Threat Behav 1994: 24: 1-9.

28. Ninan PT, van Kammen DP, Scheinin M, Linnoila M, BUNNEY WE, GOODWIN FK. CSF 5-hydroxyindoleacetic acid levels in suicidal schizophrenic patients. Am J Psychiatry 1984: 141: 566-569.

29. Virkunnen M, DeJong J, Bartko J, Linnolla $M$. Psychobiological concomitants of history of suicide attempts among violent offenders and impulsive fire setters. Arch Gen Psychiatry 1989: 46: 604-606.

30. Cremniter D, Thenault M, Jamain S, Meidinghir A, Delmas C, Gaillard M. Serotonin and suicide: a preliminary study concerning a sample of violent suicidal patients. Prog Neuropsychopharmacol Biol Psychiatry 1994: 18: 871-878. 